Pour faire ce canal latéral au Rhône, établi à grande section sur la rive gauche, au double point de vue de la navigation et des forces motrices, faudra-t-il dépenser 500 millions ou deux milliards?... On voit bien qu'il rendrait service au comnerce et a l'industrie, mais étant donné que l'on ne peut pas déterminer l'importance du bénéfice qui doit en résulter, il sagit avant tout de savoir, dans les limites du possible, si la rémunération de l'énorme capital nécessaire à l'entreprise est dans l'ordre des choses probables. Et pour cela il est de toute évidence qu'il faudrait d'abord savoir : $1^{0}$ si cet aménagement est possible au point de vue technique; $2^{\circ}$ quelle dépense approximative il entrainerait.

Il nous semble donc que le premier objet d'une Association d'études devrait être non pas d'agiter, à l'aventure, tous les milieux intéressés, mais de réunir un capital suffisant pour permettre à des ingénieurs compétents de travailler sur le terrain, de recueillir, niveau et boussole en mains, tous les éléments topographiques, géologiques et hydrologiques indispensables à la rédaction d'un avant-projet. Après cela on pourrait utilement discuter de la possibilité ou de l'impossibilité du problème, et si l'on reconnaît qu'il est soluble il sera temps d'agiter l'opinion pour décider les pouvoirs publics à se metre à l'ceuvre.

Note de la Rédaction.

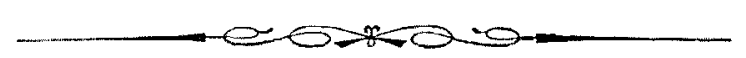

\section{CHAUDIËRE ÉLECTRIQUE}

On s'est souvent demandé si un grand nombre d'usines, telles que les fabriques de produits chimiques, les stéarinerves, les sticreries, les distilieries, les fabriques de matières colorantes, etc., etc., utilisant déjà l'électricité pour la force motrice ou l'éclairage, n'auraient pas aussi avantage a employer l'énergie électrique pour le chauffage de leurs appareils de concentration des liquides et de distillation. Si le calcul indique qu'en produisant l'énergie électrique par le charbon cette opération serait extrêmement désavantageuse, on trouve par contre, qu'avec de la houille blanche à bon marché le chauffage électrique est dans l'ordre des choses possibles.

Il ne s'agit pas, évidemment, de créer une installation hydro-électrique spécialement en vue du chauffage, mais d'employer, dans une usine alimentée par la houille blanche, une partie du courant à certaines opérations de concentration des liquides ou de distillation. L'accès du charbon aux usines hydro-électriques est généralement difficile et le prix de revient du chauffage par les procédés usuels est une dépense, toutes choses restant égales d'ailleurs, plus élevée dans ces usines que dans celles situées à proximité des centres d'approvisionnement. Ces usines hydro-électriques ont l'avantage, par leurs procédés électrochimiques, de réaliser une fabrication plus économique, mais elles perdent une partie de cet avantage par l'obligation ou elles se trouvent d'appeler la houille noire à l'aide de la houille blanche dans l'élaboration finale de leurs produits. Il serait donc extrêmement intéressant de pouvoir, dans les cas semblables, se passer completement du charbon. Pour ne citer qu'un excmple, considérons une usine produisant de la soude électrolytique. La soude caustique sort des électrolyseurs en lessive assez faible; on concentre cette lessive dans des appareils à triple-effet, ensuite on la solidifie dans des chaudrons. Or, ces deux dernières opérations exigent une dépense notable de charbon. Si l'on pouvait employer une partie de l'énergie électrique dont dispose lusine au chauffage des triple-effets - et des chaudrons de solidification ( $\mathrm{I}$ ), - et si seulement le prix de revient de ces opérations était égal à celui des procédés actuels au charbon, ce serait là déjà un grand avantage, car on n'aurait affaire qu'à un seul fournisseur d'énergie et l'on serait à l'abri de la fluctuation des cours, et des grèves toujours de plus en plus probables.

Done, si des applications de ce genre deviennent économiquement possibles, on conçoit qu'elles puissent constituer un débouché de haute importance pour la houille blanche. Bien des industries - sans parler de la métallurgie, de la verrerie - dans lesquelles le charbon employé à la préparation des produits représente une forte composante du prix de revient comparativement à la dépense de force motrice, n'ont pas de motifs de devenir des clients de l'énergie hydro-électrique; il en serait tout autrement si elles pouvaient remplacer leur charbon, sinon en totalité, du moins pour la plus grande partie, par le chauffage électrique. La question vaut la peine d'être étudiée.

Il a déjà été indiqué ici que l'on avait essayé cette application dans certaines industries chimiques, notamment à la concentration de l'acide sulfurique. Cette opération, par les procédés de chauffage usuels, est assez difficile, parce que l'on ne peut employer que des récipients en matiere inattaquable, platine ou plomb. Mais les appareils en platine sont fort coûteux et ceux en plomb devant être à parois très épaisses se prêtent mal aux échanges calorifiques. Le chauffage direct de la liqueur par un conducteur électrique immergé dans l'appareil est beaucoup plus commode à tous points de vue. Ce procédé a cependant reçu peu d'applications pratiques, parce que le prix du chauffage ainsi obtenu était élevé en raison, d'abord de la production de l'énergie électrique par le charbon, et ensuite de l'amortissement de l'installation coûteuse des appareils électrogènes. En admettant que l'énergie électrique soit achetée à une usine hydraulique, l'opération semble devoir être encore plus onéreuse que le chauffage direct au charbon, car, par de bons dispositifs, on utilise assez bien, dans ce cas, la chaleur du foyer. Il n'en est pas moins vrai que, si cette concentration devait se faire dans une usine électrochimique produisant elle-même son énergie, il y aurait probablement égalité de prix, à peu de chose près, entre les deux procédés, et les avantages du chauffage électrique feraient, croyons-nous, qu'on lui donnerait la préférence. Le simple calcul que nous indiquons plus loin permettra de se rendre compte aisément de cette égalité de prix.

Le cas où le chauffage électrique nous semble devoir être tenté est le suivant, cas qui se rencontre assez fréquemment dans beaucoup d'usines. Il s'agit de porter un liquide à

(1) Cela ne paraît pas impossible, puisqu'ainsi que nous le dirons en-un prochain article, on essaie actuellement avec succès le chauftage électrique des fours de verrerie. 
l'ébullition dans un appareil qui se trouve éloigné des générateurs de vapeur qui alimentent l'usine. Le rendement de l'appareil peut être considéré comme étant égal à 0,70 , celui du générateur à 0,60 et enfin celui de la conduite de vapeur à 0,95 . Dans ces conditions, souvent réalisées en pratique, le rendement total en énergie calorifique dans l'appareil d'utilisation sera :

$$
0,70 \times 0,60 \times 0,9^{5}=0,399 \text {, soit } 0,40 \text {. }
$$

Par contre, il existe un dispositif que nous allons décrire, permettant de chauffer directement par l'électricité cet appareil d'utilisation et avec un rendement supérieur à 95 pour roo.

Faisons le calcul du coût du chauffage dans les deux cas :

Une calorie correspondant à un travail économique de 425 kilogrammetres, il faudra $425: 75=5,67$ chevaux pour développer une calorie par seconde, ce qui revient à dire qu'en une heure ces 5,67 chevaux produiront théoriquement : $60 \times 60=3600$ calories.

Si nous prenons de la houille de moyenne qualité, c'està-dire donnant 7000 calories par kilogramme, nous voyons que ces 3600 calories n'exigeront que ok50o environ.

Considérons maintenant les rendements :

Pour le chauffage au charbon il faudra dépenser effectivement, pour produire ces 3600 calories:

$$
o^{k} 500 \frac{100}{40}=1 \mathrm{k} 250
$$

Pour le chauffage électrique la puissance effective aux bornes de l'appareil d'utilisation sera :

$$
5,67 \frac{100}{9^{5}}=5,97 \text { soit } 6 \text { chevaux. }
$$

Comptons le charbon à 30 francs la tonne, rendu dans le foyer du générateur, et le cheval-an électrique à 40 francs (prix de revient). ( 1 )

Le chauffage au charbon coûtera: $0,03 \times 1,250=0^{f} 0375$, et le chauffage électrique, en comptant 7600 heures seulement de marche dans l'année :

$$
\frac{40}{7600} \times 6=\text { of } 0316
$$

On voit donc, toute approximation gardée, que l'écart n'est pas sensible entre les deux procédés et qu'en étudiant la question de pres on trouverait souvent avantage, dans des cas analogues, à employer le chaufiage électrique.

L'appareil répondant aux considérations de ce calcul est une petite chaudière électrique, très simple comme construction et fonctionnement, dont un modèle, dû à M. Ougrimoff, professeur à l'Ecole impériale polytechnique de Moscou, figurait a l'Exposition universelle de Paris en 190o. Nous donnons, ci-joint, un dessin effectué d'après croquis montrant les dispositions essentielles de l'appareil. A notre connaissance il n'a encore été décrit que dans le

(I) C'est évidemment ce prix seul qu'il importe de considérer puisque l'énergie électrıque remplace ici le charbon qui entre dans la fabrication et sur le prix duquel on ne fait pas porter de bénéfice.

Ce prix de revient de 40 francs le cheval-an est, nous assure-ton, abtenu dans plusieurs installations.
${ }_{11}$ e fascicule (Electrochimie) de L'Electricité à l'Expo. sition de rgoo rédigé par MM. Montpellier, Bainville et Brochet. Les données économiques sur lesquelles sont, dans ce livre, établis les prix comparatifs du chauffage à vapeur usuel et du chauffage par la chaudière Ougrimoff nous semblent trop favorablement choisies pour faire ressortir la supériorité de cet appareil. Quoiqu'il en soit, il semble susceptible d'emploi, ainsi que le montrent encore nos calculs moins avantageux pour lui et, à ce titre, nous avons cru intéressant de le signaler ici.

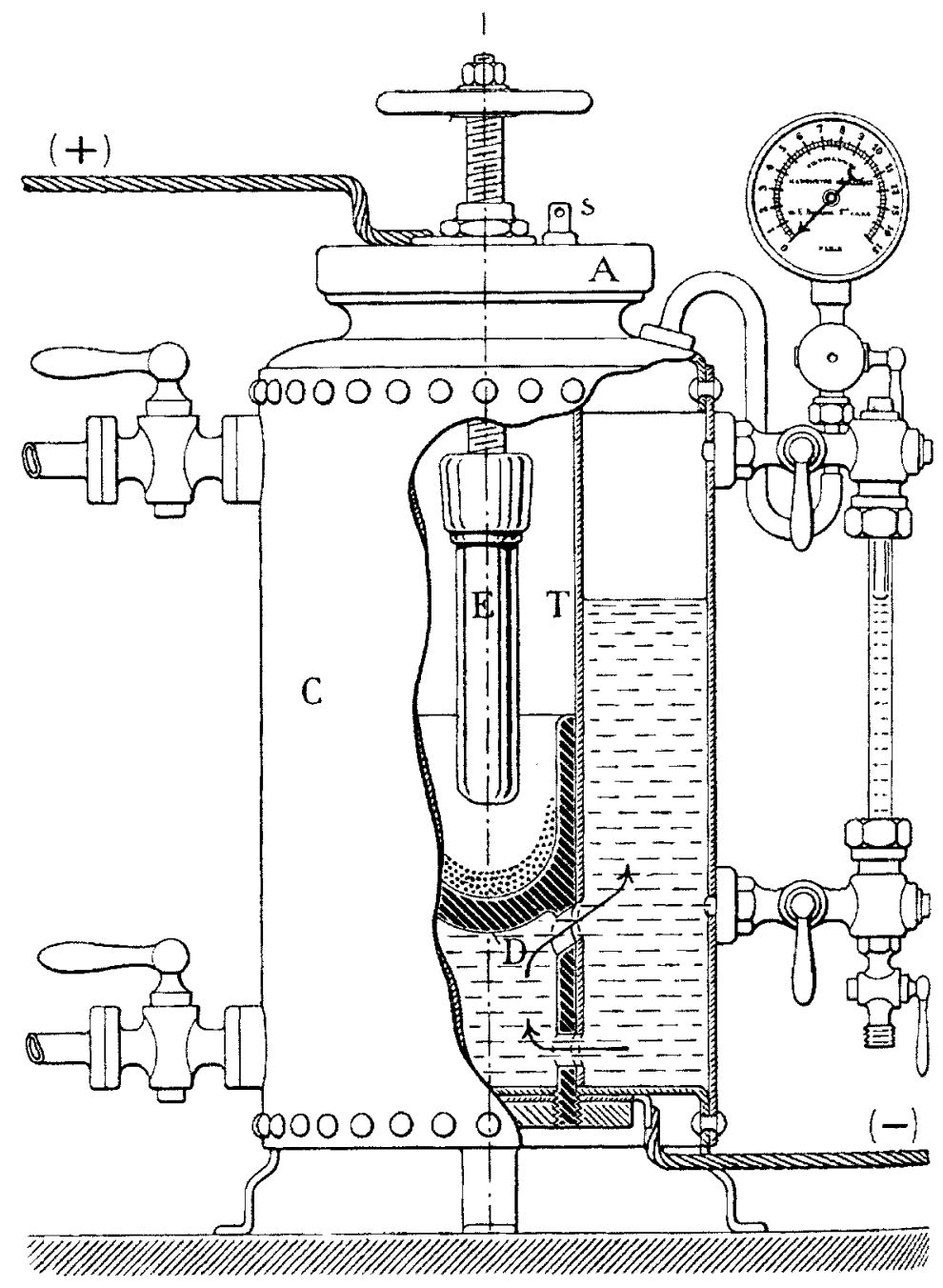

Chaudière. Electrique de M. Ougrimoff

La chaudière présentée par $M$. Ougrimoff était formée d'un corps cylindrique vertical $\mathrm{C}$, en tôle, de 20 centimètres de diamètre extérieur sur environ 40 centimètres de halteur, à l'intérieur duquel était placé concentriquement et sur toute sa hauteur un tube de fer T hermétiquement clos; c'est le tube de chauffe. Il était garni d'un manchon intérieur en fonte formant, en D, une cuvette à parois assez épaisses sur lesquelles était appliquée une garniture en poudre de graphite comprimée, laquelle constituait l'un des pôles de l'appareil de chauffage. L'autre pôle était une électrode $\mathrm{E}$ en charbon, portée par une tige filetée se dépla. çant dans un écrou soigneusement isolé dans un disque $A$ non conducteur, fixé sur le couvercle de la chaudière. C'est entre ces pôles dont la distance était ainsi réglable qué l'on faisait jaillir l'arc électrique; il se produisait doncelf vase clos. Une petite soupape $s$, disposée sur le couverde, permettait le dégagement des gaz du tube de chauffe all 
moment de la mise en marche et s'opposait aux rentrées d'air pendant le fonctionnement.

Des càbles métalliques mettaient la masse de la chaudière et, par conséquent, la cuvette D au pôle négatif du courant, et la tige filetée et l'électrode $\mathrm{E}$ au pôle positif.On comprend aisément que c'est dans ce sens que devait se faire la nise en circuit de l'appareil. Le charbon graphitique pulvérulent entrainé dans l'arc, passait de l'électrode $\mathrm{E}$ sur la couche de graphite de la cuvette $\mathrm{D}$, ce qui compensait largement l'usure de l'électrode négative par suite de la combustion de ses particules dans l'arc.

L'ípaisseur de cette garniture se maintenait donc sensiblement constante, ce qui était d'une assez grande importance, au point de vue de la durée et du bon fonctionnement du tube de chauffe. La couche de graphite avait pour but et de protéger les parois métalliques contre la température de l'arc, et de servir de résistance au démarrage, à la mise en marche. Froide, la poudre de graphite présente une résistance snffisante pour que, lorsqu'on amène au contact deux électrodes afin d'amorcer l'arc, il ne se produise pas entre elles de court-circuit. Au fur et à mesure que la température du graphite s'élève, sa résistance diminue et on règle alors la distance des électrodes suivant l'arc que l'on veut obtenir. Le réglase de l'appareil qui nous occupe était, comme on le conçoit, très simple et ne nécessitait pas une grande précision; on aurait pu le rendre automatique.

Cette petite chaudière, produisant de la vapeur à 7 kilos de pression fonctionnait avec un courant de 100 ampères sous une tension de 80 volts en moyenne. Son rendement était, nous dit-on, de $97 \%$ environ. Elle possédait tous appareils accessoires nécessaires: manomètre, niveau d'eau, soupape, alimentation.

Nous ignorons si, depuis l'Exposition, M. Ougrimofl a expérimentć de plus grands modèles, ou si l'on a construit sur ce principe d'autres types de chaudières électriques. Cela est fort possible.

Si la chaudière électrique, en tant que générateur proprement dit de vapeur n'est pas intéressante, il peut en être différemment pour certains cas particuliers, quand, par exemple, dans une usine où la houille blanche est à bon marché, il s'agit d'appareils à concentration des liquides ou de distillation, éloignés d'un générateur de vapeur et à fonctionnement intermittent.

Il nous semble que le dispositif du professeur russe peut recevoir quantité de variantes, voire même de perfectionnements s'ils n'existent pas déjà et, par suite, se prêter à de nombreuses applications industrielles. Ici, comme dans la plupart des autres applications du courant électrique, l'arantage de pouvoir fractionner l'énergie au gré de toutes les nécessités nous semble encore un argument en faveur de ce mode de chauffage.

La verreric et la métallurgie entre autres, cherchent à s'affranchir du charbon en demandant au courant électrique seul toute l'énergie dont elles ont besoin; elles $y$ arriveront. Souhaitons donc aussi que les usines de produits chimiques qui emploient déjà la houille blanche arrivent, en même temps, à se passer de l'autre pour l'élaboration finale de leurs produits.

$$
\text { E.-F. Côte. }
$$

\section{LE MOIS HYDR0-ÉLECTRIQUE \\ en France et à l'Etranger}

\section{INFORMATIONS DIVERSES}

\section{Projet général des installations de la «Société Electrochimique de la Romanche p pour le service de la ville de Grenoble.}

L'ensemble des installations que la Société Electrochimique de la Romanche va exécuter pour l'alimentation en énergie électrique de la ville de Grenoble, et dont nous donnerons ultérieurement des plans de détail, se compose, en principe :

so De l'usine génératrice de Livet, qui comprendra: 3 groupes électrogènes à courants triphasés à 3500 volts de 2500 chevaux chacun; 3 transformateurs-élévateurs de 3500:26000 volts, de $2500 \mathrm{~K}$.V.A. chacun; 2 groupes électrogènes à courant continu à 150 volts de $17^{5}$ chevaux chacun, pour l'excitation et les services accessoires.

$2^{\circ}$ De la ligne Livet-Grenoble.

3o De 3 transformateurs-abaisseurs à installer dans l'usine municipale réceptrice de la ville de Grenoble; ils abaisseront le voltage de 26000 volts à 5000 et seront chacun d'une puissance de $2500 \mathrm{~K}$.V.A.

Dès le début on n'installera que : 2 groupes générateurs de 2500 chevaux, 2 groupes d'excitation de 175 chevaux, 2 iransformateurs-élévateurs $2500 \mathrm{~K}$.V.A. et la ligne de Livet-Grenoble complète. (L'un des appareils de chaque catégorie assurera le service tandis que l'autre servira de réserve). Les aumes appareils ne seront installés que lorsque le service de la ville exigera plus de 2000 chevaux à la porte Mallifaud.

L'exéculion des turbines est confice à la maison A. et $\mathrm{H}$. Bouvier, de Grenoble; celle du matériel électrique et de la ligne de transport, à la Cumpagnie Electro-Mécanique BrownBoveri: la construction des bâtiments à $M$. Fayolle, entrepreneur de la ville de Grenoble.

Turbines. - $I^{\circ}$ Les turbines de 2500 chevaux sont à axe horizontal et capables de fournir chacune cette puissance dis. ponible sur l'arbre, à la vitesse de 375 tours par minute, ainsi qu'à toutes les vitesses de $10 \%$ supérieures ou inférieures a 375 tours. Elles sont du type centripète à réaction, avec aspiration et fonctionneront sous une chute totale de 57 mètres pouvant se réduire à 53 mètres environ avec les pertes de charge.

Elles sont munies chacune d'un servo-moteur hydraulique fonctionnant avec la pression de l'eau de la chute. La soupape de ce servo-moteur pourra être commandée soit à la main, soit par le régulateur automatique. Elles comprennent aussi des compensateurs de débit évitant les coups de bélier et des limiteurs de vitesse agissant en cas d'emballement. Elles peurent résister pendant deux minutes à l'emballement total (le régulateur et les limiteurs de tensic n étant supprimés), jusqu'à 670 tours.

Leur rendement garanti est au minimum de $: 0,78$ à pleine charge; 0,74 a $3 / 4$ de charge; $0,6+4$ à $1 / 2$ charge et 0,35 à $1 / 4$ de charge.

L'eau utilisée sera mesurée à l'aide d'un déversoir en mince paroi ; les pressions au manomètre et à l'indicateur de vide; la puissance recueillie par le rendement des machines électriques.

Le régulateur central se compose d'une pompe à croix commandée par un moteur électrique synchrone actionnant 